\title{
"Mistletoe sign": probably a new dermoscopic descriptor for melanoma in situ and melanocytic junctional nevus in the inflammatory stage
}

\author{
Grażyna Kamińska-Winciorek ${ }^{1}$, Paweł Właszczuk ${ }^{2}$, Jerzy Wydmański ${ }^{13}$ \\ ${ }^{1}$ Center for Cancer Prevention and Treatment, Katowice, Poland \\ Head of Department: Beata Wydmańska \\ 2Department of Pathomorphology, Medical University of Silesia, Katowice, Poland \\ Head of Department: Maciej Kajor MD, PhD \\ ${ }^{3}$ Department of Conventional and Intraoperative Radiotherapy, Maria Skłodowska-Curie Memorial Cancer Centre and Institute \\ of Centre Oncology, Gliwice Branch, Gliwice, Poland \\ Head of Department: Leszek Miszczyk MD, PhD
}

\begin{abstract}
Melanomas in situ (MIS) are difficult to diagnose as they lack well-established, dermoscopic descriptors. In numerous clinical cases, there are no definitive differentiating criteria between atypical nevus and melanoma in situ. So far, no digital dermoscopic criteria have been developed which can clearly distinguish atypical naevi from MIS. It is necessary to search for predictors of MIS and clinically suspected skin lesions in dermoscopy. We present 2 patients diagnosed with and treated for melanoma in situ and junctional nevus in its inflammatory stage. This includes a new morphological structure in dermoscopy known as the "mistletoe sign". Below, we have described dermoscopic pictures, with appropriate histopathology, for patients with the "mistletoe sign". Dermoscopy in two cases revealed multiple, well-circumscribed areas, consisting of non-uniform, sometimes pseudo-dichotomously branched structures, mimicking pseudopods, which were not reticular, arising from overall reticular or homogenous patterns resembling the mistletoe. Due to the fact that this is one of the several reports of its kind, further research and observation are still necessary. The "mistletoe sign" may be a descriptor of the melanocytic nevus in the inflammatory stage and the melanoma in situ; however, further studies are necessary.
\end{abstract}

Key words: dermoscopy, dermatoscopy, melanoma in situ, melanocytic nevus, mistletoe sign.

\section{Introduction}

Melanomas in situ (MIS) are difficult to diagnose as they lack any well-established, dermoscopic descriptors [1]. We present a new alert mnemonic descriptor of clinically suspected, pigmented lesions, described as the "mistletoe sign". In many clinical cases, there are no definitive differentiating criteria between the atypical nevus and the melanoma in situ. Yet, in 2005, Burroni et al. [1] concluded that there have, as yet, been no digital dermoscopic criteria which can clearly distinguish atypical naevi from MIS [1]. What is more, a previous study of Pizzichetta et al. [2] did not allow to distinguish melanoma in situ from the invasive type of melanoma (ICM), based on dermoscopy [2]. The dermoscopic criteria for MIS were similar to those for ICM, although white scar-like areas and linear and/or dotted vascular patterns were observed, only in patients with ICM [2].

We present the "mistletoe sign" as a new dermoscopic descriptor of clinically suspected melanocytic lesions in dermoscopic and histopathological aspects.

\section{Case reports}

In two presented cases, clinical and dermoscopic examination was performed during routine dermatological consultation. All dermoscopic pictures were examined using a non-polarized dermoscope with USG gel immersion. The dermoscopic features were described based

Address for correspondence: Grażyna Kamińska-Winciorek MD, PhD, Center for Cancer Prevention and Treatment, 16 Fliegera St, 40-960 Katowice, Poland, fax/cell phone: +48698626 208, e-mail: dermatolog.pl@gmail.com Received: 21.04.2013, accepted: 20.08.2013. 
on digital overview images which were electronically stored by a mobile digital dermoscopy imaging system (Handyscope dermoscope).

\section{Cases on dermoscopy and histopathology}

We present 2 patients diagnosed with and treated for melanoma in situ and junctional nevus in its inflammatory stage, which include a new morphological structure on dermoscopy called the "mistletoe sign".

Case 1. A 27-year-old female patient was admitted to the Dermatologic Outpatient Clinic due to the appearance of a lesion, which had been present on the skin for a period of 2 years. The pigmented lesion was located on the front of the right thigh and rapid changes within it, such as enlargement and changes in color were noted. No clinical observations of any inflammation had been noted. On dermoscopy, in the upper parts of the lesion (at 9.00 and 14.00 o'clock) two roundish clusters resembling the mistletoe were noted, with a newly-developing cluster in the lower part of the lesion (at 6.00 o'clock) (Figure $1 \mathrm{~A}$ ). In respect of the condition's history, dermoscopy and the positive "ugly duckling" sign, the lesion was entirely excised and the diagnosis of the melanocytic junctional nevus in the inflammatory stage was confirmed.

Case 2. A 37-year-old female patient was treated surgically due to a skin lesion which had been present from a period of 4 years, extremely changed in color and in structure within a period of the last 2 years. On dermoscopy of the pigmented lesion located on the internal part of the left forearm, two clusters resembling the mistletoe (at 11.00 and 14.00 o'clock) were duly noted (Figure 1 B). The histopathology of the lesion, excised in its entirety, pointed to the melanoma in situ diagnosis.
Dermoscopy in the case of these two patients revealed a number of multiple, well-circumscribed areas, consisting of non-uniform, sometimes pseudo-dichotomously branched structures, resembling pseudopods, which were not reticular, arising from an overall reticular or homogenous pattern (Figures 1 A, B), resembling the mistletoe (Figure 1 C).

All histopathological diagnoses in the reported patients were standardized according to the WHO classification. The materials concerning the 2 patients (case 1 and case 2 ) were duly compared with each other. The slides were stained with $\mathrm{H}+\mathrm{E}$, and examined under a magnification of 40 to $400 \times$. In the first case, the histological examination confirmed the diagnosis of the melanocytic junctional nevus in its inflammatory stage. Intraepidermal nests of nevus cells were found along the dermo-epidermal junction - characteristic of the so-called junctional activity. The cells were oval to cuboidal in shape, with clear cytoplasm, containing a variable amount of melanin pigment. Mitoses were rare or absent. Nests of nevus cells sometimes bulged into the underlying dermis, which may have contained a small number of melanophages and an abundance of lymphohistiocytic infiltrates (Figures $2 \mathrm{~A}, \mathrm{~B}$ ).

In case 2 , the melanoma in situ was diagnosed histopathologically. The tumour was comprised of large, neoplastic melanocytes, which extended among the keratinocytes into a "buckshot scatter" or a pagetoid pattern. Under high-power magnification, the malignant cells displayed eccentrically placed nuclei, melanin pigment in the cytoplasm, nuclear polymorphism and large nucleoli, with irregular contours. Mitoses were not frequent. The melanocytes may be distributed either singly, or in the form of nests. In MIS, the stroma and inflammatory reaction tend to be inconspicuous and may, in certain cases, be absent (Figures $3 \mathrm{~A}, \mathrm{~B}$ ).

\section{A}

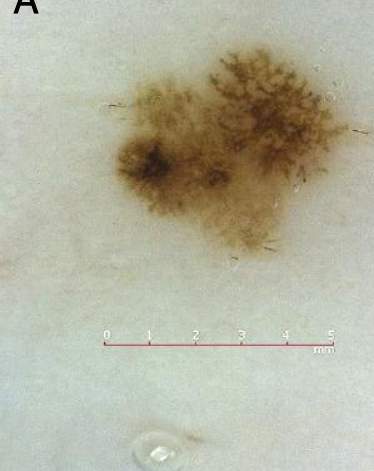

\section{B}

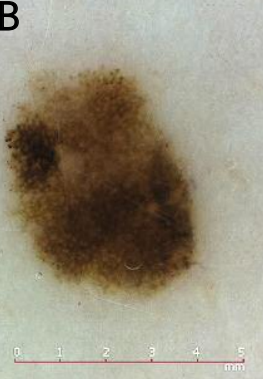

Figure 1. A - Dermoscopy. Case 1: Right upper thigh, diameter $5 \mathrm{~mm}$; melanocytic junctional nevus in the inflammatory stage. In the upper parts of the lesion (at 9.00 and 14.00 o'clock), two roundish clusters resembling the mistletoe had been observed, also a new developing cluster in the lower part of the lesion (at 6.00 o'clock). B - Dermoscopy. Case 2: Left internal forearm, diameter $4 \mathrm{~mm}$; superficial melanoma spreading type (in situ). In the upper parts of the lesion (at 11.00 and 14.00 o'clock), two clusters resembling the mistletoe had been noted, more concentrated than the cases described above. C - The mistletoe on the tree as a well-visible, multiple, clustered, globular plant, in the shape of a woody shrub; please note the typical, pseudo-dichotomously branched structures 

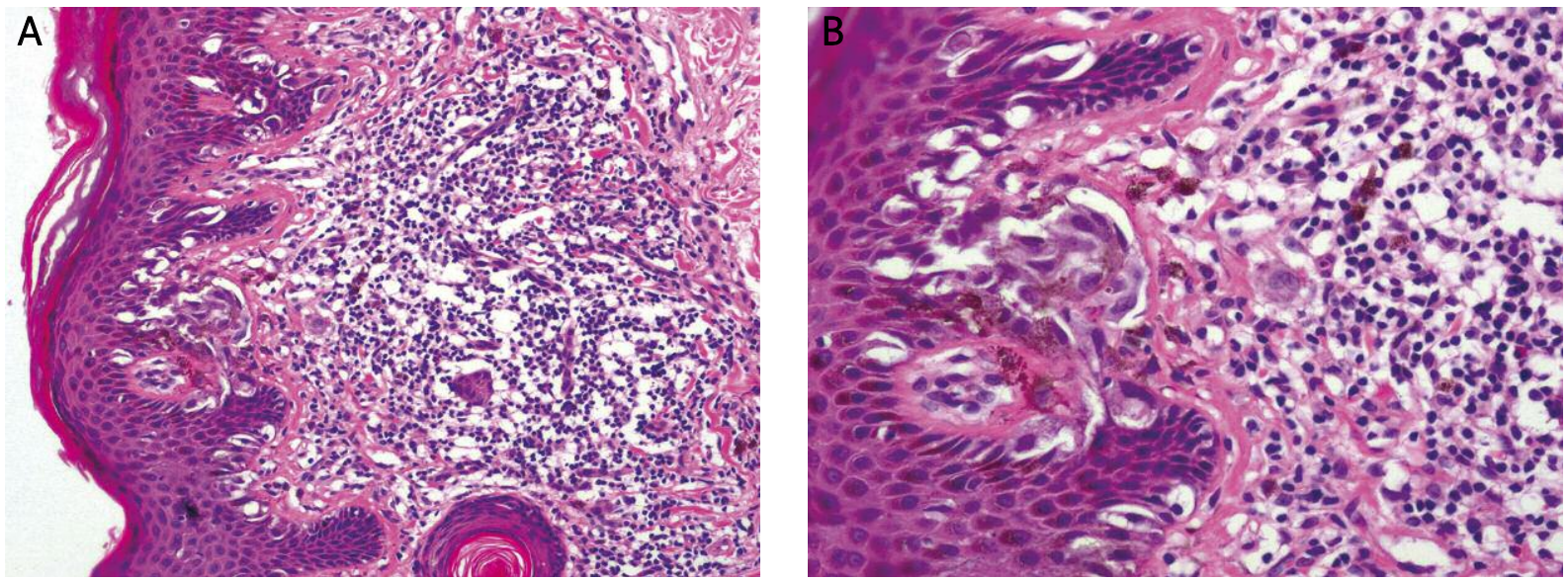

Figure 2. A - Histopathology. Case 1: Melanocytic junctional nevus in its inflammatory stage; $\mathrm{H}+\mathrm{E}$ stain, magnification 100 x. B - Case 1: Melanocytic junctional nevus in its inflammatory stage, intraepidermal nests of nevus cells were found along the dermo-epidermal junction; $\mathrm{H}+\mathrm{E}$ stain, magnification $200 \times$
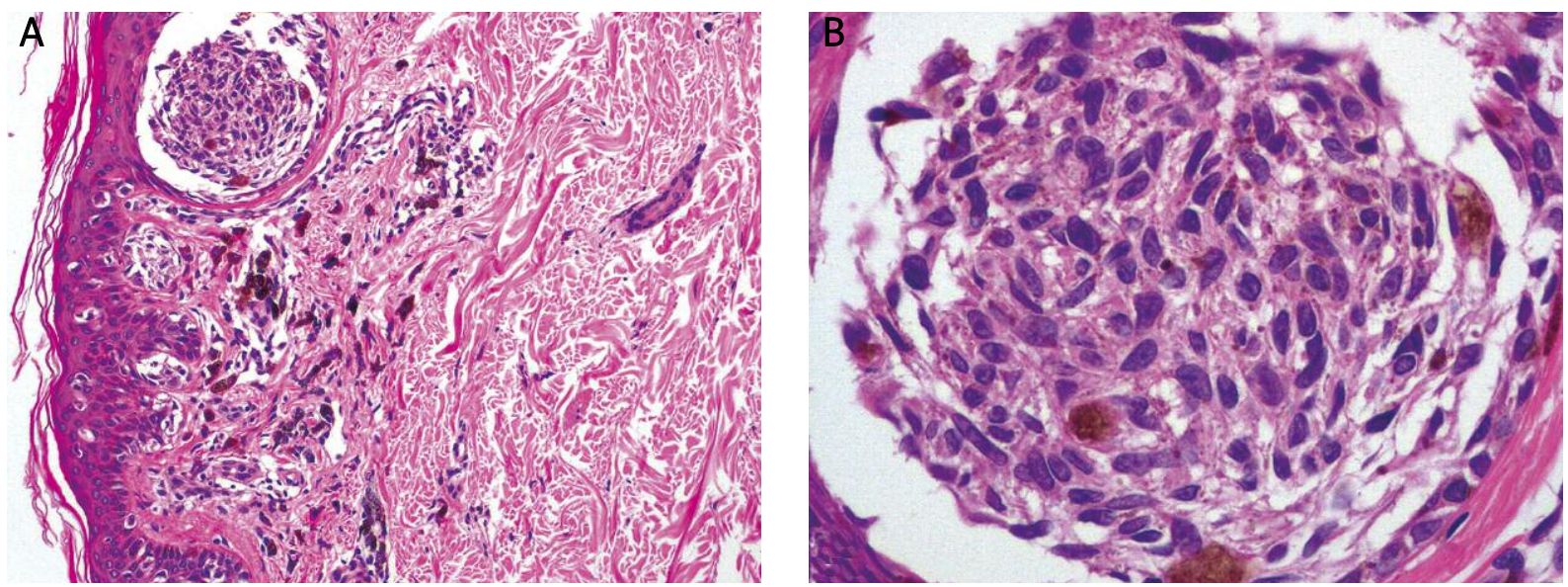

Figure 3. A - Histopathology. Case 2: Melanoma in situ; H + E magnification 100×. B - Case 2: Melanoma in situ, nests of melanoma cells; $\mathrm{H}+\mathrm{E}$ stain, magnification $400 \times$

\section{Discussion}

In accordance with the latest available data, with respect to atypical naevi, MIS more frequently appears larger in size, with an atypical network spreading over more than half of the lesion, the contemporary presence of more than one type of network and more extended reticular grey-blue regression areas located both in the centre and at the periphery of the lesion [3]. In the latest report by Seidnari et al. [4], eight different dermoscopic subtypes of MIS were duly distinguished. The most common ones were as follows: reticular grey-blue (27.2\%), reticular (21.1\%), multicomponent (20.2\%) and those rarely occurring: island (10.5\%), spitzoid (7\%), the inverse network (6.1\%), "net-blue globules" (5.3\%) and globular (2.6\%). Previously Borsari et al. [5] concluded that the dermoscopic island is a highlysensitive melanoma in situ predictor. The dermoscopic island was defined as a well-circumscribed area, presenting a uniform dermoscopic pattern which differs from the remainder of the pigmented lesion [5]. Due to the fact that the dermoscopic island was present in $10.4 \%$ of in situ melanomas, in $3.1 \%$ of atypical dermoscopic nevi and only in the case of $4.1 \%$ of invasive melanomas, this descriptor is considered, according to Borsari et al. [5], to be a potential early sign of transformation of a nevus into a melanoma. Among all the dermoscopic descriptors of the melanoma, including the MIS, the "mushroom-cloud sign" [6] is also mentioned. This term represents the hyperpigmented area which has extended in one direction, beyond the border of the lesion, and the plume underneath represents the stalk-like projection [6]. According to Bowling [7], the presence of irregular streaks at one polar end, eccentric hyperpigmentation as a combination of eccentric atypical network, irregular globules and blotches may also be seen in invasive melanomas as a lesion of no more than $5 \mathrm{~mm}$ in size [7]. 
We propose the term "mistletoe sign" as a new descriptor of the melanoma in situ and the inflammatory melanocytic junctional nevus. Probably the "mistletoe sign" was a first alert symptom of abnormal melanocytic proliferation shown on dermoscopy of junctional nevus in the inflammatory stage. The mistletoe, known as the Viscum album, is a small, woody shrub, frequently globular in shape, which grows on the branches of other trees, with the stems appearing characteristically forked (pseudo-dichotomously branched) [8] (Figure 1C). The "mistletoe sign" represents multiple, well-circumscribed areas, consisting of nonuniform, at times pseudo-dichotomously branched structures, resembling pseudopods, which are not reticular, arising from an overall reticular or homogenous pattern (Figures 1 A, B). This term combines previously described, well-known features of melanoma in situ such as the island and the spitzoid [4], however this is not solely typical of any of these. Recent data concerning Polish patients published by Czerwinska et al. [9] showed that most of the lesions were diagnosed at Clark IV and Clark III, Breslow IV level. Melanoma is highly treatable in its earlier stages; hence there is a great necessity of public education of the early signs of melanoma, which could improve the accuracy of skin self-examination, and would allow the targeted interventions of dermatologists to be successful [9].

We believe that the "mistletoe sign" is an alert mnemonic descriptor which will be useful in screening and follow-up treatment of all pigmented lesions which have been administered before the facilitation of dermoscopy, especially those clinically suspected. In both cases, the presence of the "mistletoe sign" led to the excision of the suspicious lesions.

Due to the fact that this is one of the several reports of its kind, further research and observation are still necessary.

\section{References}

1. Burroni M, Sbano P, Cevenini G, et al. Dysplastic naevus vs. in situ melanoma: digital dermoscopy analysis. Br J Dermatol 2005; 152: 679-84.

2. Pizzichetta MA, Argenziano G, Talamini R, et al. Dermoscopic criteria for melanoma in situ are similar to those for early invasive melanoma. Cancer 2001; 91: 992-7.

3. Seidenari S, Ferrari C, Borsari S, et al. The dermoscopic variability of pigment network in melanoma in situ. Melanoma Res 2012; 22: 151-7.

4. Seidenari S, Bassoli S, Borsari S, et al. Variegated dermoscopy of in situ melanoma. Dermatology 2012; 224: 262-70.

5. Borsari S, Longo C, Ferrari C, et al. Dermoscopic island: a new descriptor for thin melanoma. Arch Dermatol 2010; 146: 1257-62.

6. Mahlberg MJ, Hwa C, Kopf AW, Stein JA. "Mushroom-cloud sign" of melanoma. Dermatol Surg 2011; 37: 1546-8.

7. Bowling J (ed.). Diagnostic dermoscopy. The illustrated guide. Willey-Blackwell, Oxford 2012.

8. http://www.kew.org/plants-fungi/Viscum-album.htm
9. Czerwińska M, Alekseenko A, Rup E, et al. Retrospective data analysis of the history of patients treated for malignant melanoma at the Department of Dermatology, Jagiellonian University between 1991 and 2008. Postep Derm Alergol 2011; 28: 92-6. 